\title{
Combined measurements of tumor number and size helps estimate the outcome of resection of Barcelona clinic liver cancer stage B hepatocellular carcinoma
}

Xin Wang ${ }^{1}$, Zusen Wang ${ }^{1}$ and Liqun $\mathrm{Wu}^{1,2^{*}}$

\begin{abstract}
Background: Although the Barcelona Clinic Liver Cancer (BCLC) staging system suggests that patients with stage B hepatocellular carcinoma (HCC) should be treated with transcatheter arterial chemoembolization instead of surgical treatment, recent studies indicated that the prognosis of surgical resection for patients with BCLC stage B HCC was better than that of TACE. However, the portion of patients with stage B that will achieve better outcomes from surgical treatment remains unclear. In this study, we identified risk factors that influence the prognosis of BCLC stage B HCC after RO surgical resection to determine whether some patients with stage B HCC may benefit more from $\mathrm{RO}$ resection than other patients and to provide a guideline to estimate the tendency.
\end{abstract}

Methods: The clinical data of 78 patients with BCLC stage B HCC after RO surgical treatment within 11 years were analyzed retrospectively, using relapse or death as the endpoint. Kaplan-Meier survival and Cox regression analyses were used to study prognosis (disease-free survival, DFS and overall survival, OS) and independent risk factors.

Results: For all stage B patients, 1-, 2-, and 5-year DFS rates were $62.5,36.4$, and $16.6 \%$, respectively. Cumulative tumor size $>5.0 \mathrm{~cm}$ and tumor number $\geq 4$ were independent prognostic risk factors for DFS. The 1-, 2-, and 5- year DFS rates and OS rates of patients with at least one of these two factors were 49.0, 17.2, and $7.4 \%$ (for DFS), and 78.6, 54.8, and $13.4 \%$ (for OS), respectively, which were significantly lower than patients without these two factors (77.8, 58.3, and $27.2 \%$ for DFS, and $94.4,83.3$,and $51.8 \%$ for OS, respectively, $P<0.01$ ).

Conclusions: The analyses indicated that the outcomes of RO resection were much better for patients with BCLC stage B HCC with two or three tumors and cumulative tumor sizes of $\leq 5.0$ but $>3.0 \mathrm{~cm}$ than other patients with stage B.

Keywords: BCLC staging, Hepatocellular carcinoma, Prognosis, R0 surgical treatment, Risk factors

\section{Background}

Hepatocellular carcinoma (HCC) is one of the five most common malignancies worldwide. Each year, more than 690,000 people die from liver cancer, and the number is increasing every year [1]. HCC is also the third most frequent cause of tumor-related death [2]. The clinical staging system of $\mathrm{HCC}$ has an important function for selecting treatment modes and evaluating prognosis, and in recent years, the Barcelona Clinic Liver Cancer (BCLC)

\footnotetext{
*Correspondence: wulq5810@126.com

1 Department of Hepatobiliary and Pancreatic Surgery, Affiliated Hospital of Qingdao University, Qingdao 266000, China

${ }^{2}$ Jiangsu Road 16, Qingdao 266000, China
}

staging system has received more attention. According to the BCLC staging system, only patients with very early stage and stage A (early stage) HCC should undergo surgical resection, ablation, or liver transplantation. Patients with stage B HCC should be treated with transcatheter arterial chemoembolization (TACE) [3]. However, many studies have indicated that for patients with BCLC stage $B$ $\mathrm{HCC}$, the prognosis of surgical resection is better than that of TACE [4-7], indicating that it is feasible and applicable for some patients with BCLC stage B HCC to undergo R0 section. However, the portion of patients with stage B that will achieve better outcomes from surgical treatment remains unclear. Moreover, the standard 
indicators or influencing risk factors for selection of HCC patients with stage B toward surgical resection have not yet been established. Therefore, study of the risk factors for prognosis of BCLC stage B after surgical treatment has profound significance in forming a guideline for clinical treatment of this sort of patients. Clarification of major factors influencing curative efficiency will enable clinicians to estimate whether a patient with BCLC stage B HCC may achieve a better prognosis from $\mathrm{R} 0$ resection and identification of those patients with non-improved outcomes should be more carefully monitored after resection. This study aims to provide surgeons with a guideline to estimate clinical treatment and predict prognosis to guide postoperative treatment.

\section{Methods}

\section{Case data}

From 2001 to 2012, 827 patients with HCC underwent R0 hepatic resection in the Affiliated Hospital of Qingdao University, China and 78 patients suffered BCLC stage B HCC disease. We declare that informed consents for the use of their clinical data in this study were obtained from all participants or their next of kin before follow up and before the study began. We thank them a lot for their consent of this study. BCLC staging was determined using liver ultrasonography or computed tomography (CT) and thorough clinical assessment. All the patients had 2-5 cancerous nodules with a cumulative tumor size of $>3.0 \mathrm{~cm}$, a ChildPugh score of $A-B$, and performance status of 0 , and thus, were classified as BCLC stage B. Pathological diagnoses were made for all patients. There were 66 men and 12 women (ratio 5.5:1) and their age ranged 33-80 years old, with an average of 55.9 years. The clinicopathological features of the 78 patients are shown in Table 1 . The collection of clinical data was approved by the Ethic Committee of Affiliated Hospital of Qingdao University.

\section{Surgical methods}

All 78 cases underwent liver resection in accordance with the Couinaud segmentation method to implement hepatic segmentectomy or combined resection for adjacent liver segments (anatomical resection) or partial hepatectomy containing tumor (nonanatomical resection). R0 hepatic resection means there were no residual cancer cells present in the surgical margin when checked

Table 1 Clinicopathological features of 78 patients with Barcelona Clinic Liver Cancer stage B hepatocellular carcinoma

\begin{tabular}{|c|c|}
\hline Factors & Median (range or proportion) \\
\hline Sex (male/female) & $66: 12(84.6 \%: 15.4 \%)$ \\
\hline Age (years) & $56.0(33-80)$ \\
\hline Alcohol consumption (no/yes) ${ }^{a}$ & $56: 20(73.7: 26.3)$ \\
\hline Preoperative TACE (no/yes) & $67: 11$ (85.9 \%:14.1\%) \\
\hline HBsAg (negative/positive) & 7:71 (9.0 \%:91.0 \%) \\
\hline Anti-HCV (negative/positive) & $76: 2$ (97.4 \%:2.6 \%) \\
\hline $\operatorname{ALT}(U / L)$ & $45.5(12-191)$ \\
\hline GGT (U/L) & $49.5(15-395)$ \\
\hline Child-Pugh Classification (A/B) & $76: 2$ (97.4 \%:2.6 \%) \\
\hline Cirrhosis (no/yes) & 5:73 (6.4 \%:93.6 \%) \\
\hline Portal hypertension (no/yes) & $65: 13(83.3 \%: 16.7 \%)$ \\
\hline Liver resection range (hepatic segment) & $2(1-4)$ \\
\hline Resection margin $(\mathrm{mm})^{\mathrm{a}}$ & $5(0-30)$ \\
\hline Anatomical resection (yes/no) & $18: 60(23.1 \%: 76.9 \%)$ \\
\hline Hepatic inflow occlusion (no/yes) & $33: 45(42.3: 57.7)$ \\
\hline Intraoperative blood loss (mL) & $300(100-3500)$ \\
\hline Blood transfusion $(\mathrm{mL})$ & $0.0(0-1100)$ \\
\hline Preoperative alpha-fetoprotein ( $\mu \mathrm{g} / \mathrm{L})$ & $17.8(1.1-1211.0)$ \\
\hline Cumulative tumor size $(\mathrm{cm})$ & $5.0(2.5-20.0)$ \\
\hline Tumor number & $3 N$ \\
\hline Differentiation (high/middle and low/necrosis) & 3:73:2 (3.8 \%:93.6 \%:2.6 \%) \\
\hline Liver capsule invasion (no/yes) & $12: 66$ (15.4 \%:84.6 \%) \\
\hline
\end{tabular}

ALT alanine aminotransferase, GGT gamma glutamyltransferase, $H B s A g$ hepatitis B surface antigen 
microscopically and no tumor visible with the naked eyes.

\section{Follow-up study}

Serum alpha-fetoprotein, liver function tests, liver ultrasonography or CT and lung CT scan were performed monthly for 3 months after surgical resection. The time of recurrence after resection was adjudged by the presence of clear masses on imaging examination. The follow-up ended on 31 December 2015 or when patients died. The median follow-up time was 31.4 months (ranging 3.3113.2 months).

\section{Statistical analysis}

All the data were statistically analyzed with SPSS Statistics software for Windows, Version 13.0 (SPSS Inc., Chicago, IL, USA). The Kaplan-Meier survival analysis (log-rank test) was used to analyze disease-free survival (DFS) and overall survival (OS). The factors with $P<0.05$ were enrolled in the Cox regression hazard model. $P<0.05$ was considered significant.

\section{Results}

Risk factors for DFS and OS

Seventy-eight patients with BCLC stage B HCC underwent R0 hepatic resection. The OS rates for 1,2 and 5 years were 85.9, 67.9 and $29.8 \%$, respectively; the median OS was 35.0 months [95\% confidence interval $(C I)=29.4-40.6$ months]. The DFS rates for 1,2 and 5 years were $62.5,36.4$ and $16.6 \%$, respectively; and the median DFS was 15.0 months $(95 \% \mathrm{CI}=10.7$ 19.3 months).

In Table 2, we analyzed the relation of selected factors with the DFS with Kaplan-Meier analysis. The result showed that cumulative tumor size $>5.0 \mathrm{~cm}$ and tumor number $\geqq 4$ were important risk factors that significantly influenced DFS $(P<0.01)$. Then the two factors were enrolled into Cox regression hazard model analysis which showed that the cumulative tumor size and tumor number were two significant independent risk factors for DFS $(P<0.05)$.

\section{Relationship between recurrence and tumor number}

Patients with BCLC stage B HCC were divided into three groups according to tumor numbers which included 2, 3

Table 2 Factors influencing disease-free survival (DFS) for patients with Barcelona Clinic Liver Cancer stage B hepatocellular carcinoma after $\mathrm{R} 0$ resection

\begin{tabular}{|c|c|c|c|c|}
\hline \multirow[t]{2}{*}{ Factors } & \multicolumn{2}{|c|}{ Kaplan-Meier analysis } & \multicolumn{2}{|c|}{ Cox regression hazard model } \\
\hline & Median DFS (mo) & $P$ value & $\mathrm{HR}(95 \% \mathrm{Cl})$ & $P$ value \\
\hline Sex (male/female) & $15.0 / 13.0$ & 0.771 & & \\
\hline Age ( $\leq 60 />60$ years) & $15.0 / 13.0$ & 0.530 & & \\
\hline Alcohol consumption (no/yes) ${ }^{a}$ & 15.0/18.0 & 0.463 & & \\
\hline Preoperative TACE (no/yes) & $13.0 / 33.0$ & 0.058 & & \\
\hline HBsAg (negative/positive) & $8.0 / 18.0$ & 0.267 & & \\
\hline Anti-HCV (negative/positive) & 15.0/11.4 & 0.529 & & \\
\hline $\operatorname{ALT}(\leq 50 />50 \mathrm{U} / \mathrm{L})$ & 23.0/11.4 & 0.076 & & \\
\hline GGT ( $\leq 64 />64$ U/L) & 19.0/13.0 & 0.096 & & \\
\hline Child-Pugh classification (A/B) & 15.0/5.7 & 0.288 & & \\
\hline Cirrhosis (no/yes) & $25.0 / 15.0$ & 0.317 & & \\
\hline Portal hypertension (no/yes) & $15.0 / 23.0$ & 0.769 & & \\
\hline Liver resection range ( $\leq 2 />2$ segments) & 17.0/8.0 & 0.084 & & \\
\hline Resection margin $(<5 / \geq 5 \mathrm{~mm})^{a}$ & 13.0/19.0 & 0.573 & & \\
\hline Anatomical resection (yes/no) & $14.6 / 15.0$ & 0.783 & & \\
\hline Hepatic inflow occlusion (no/yes) & 13.0/19.0 & 0.223 & & \\
\hline Intraoperative blood loss ( $\leq 1000 />100 \mathrm{~mL}$ ) & 18.0/13.0 & 0.282 & & \\
\hline Blood transfusion (yes/no) & $17.0 / 15.0$ & 0.732 & & \\
\hline Preoperative alpha-fetoprotein $(\leq 20 />20 \mu \mathrm{g} / \mathrm{L})$ & 14.6/18.0 & 0.273 & & \\
\hline Cumulative tumor size $(\leq 5 />5 \mathrm{~cm})$ & $25.0 / 13.0$ & 0.009 & $1.685(1.011-2.808)$ & 0.045 \\
\hline Tumor number $(\leq 3 / \geq 4)$ & $23.0 / 10.1$ & 0.000 & $2.681(1.506-4.770)$ & 0.001 \\
\hline Differentiation (high/middle and low/necrosis) & 24.0/15.0/19.2 & 0.671 & & \\
\hline Liver capsule invasion (no/yes) & 13.0/17.0 & 0.882 & & \\
\hline
\end{tabular}

${ }^{\mathrm{a}}$ Some patients lacked data 
Table 3 Disease-free survival of patients with different tumor number

\begin{tabular}{lrrrrrr}
\hline $\begin{array}{l}\text { No of } \\
\text { tumors. }\end{array}$ & $n$ & $\begin{array}{l}\text { Median } \\
\text { (months) }\end{array}$ & $\begin{array}{l}\text { 1 year } \\
(\%)\end{array}$ & $\begin{array}{l}\text { 2 years } \\
(\%)\end{array}$ & $\begin{array}{l}\text { 5 years } \\
(\%)\end{array}$ & $P$ \\
\hline 2 & 39 & 28.8 & 76.9 & 53.8 & 23.1 & 0.001 \\
3 & 17 & 19.0 & 58.8 & 25.3 & 22.1 & \\
$\geq 4$ & 22 & 10.1 & 38.5 & 4.8 & 0.0 & \\
\hline
\end{tabular}

and more than 4 tumors, respectively. Kaplan-Meier survival analysis was used to analyze the DFS for the three groups. The median DFS time and the DFS rate for 1,2 and 5 years decreased as tumor number increased $(P<0.01$; Table 3$)$.

Then, the receiver operating characteristic (ROC) curve was applied for further analysis. The tumor number was regarded as a test variable while the recurrence a state variable. The area under the curve was $69.4 \%(P=0.024)$ and the Youden index was 2.5 (specificity $=78.6 \%$, sensitivity $=$ $56.3 \%$ ) (Fig. 1). We took the tumor number of 3 as the critical value according to the Youden index. The 1-, 2-, and 5-year DFS rates for the patients with tumor number $\leq$ 3 (56 cases) and $\geq 4$ (22 cases) were 71.4, 48.2, and $23 \%$ (for number $\leq 3$ ), and $38.5,4.8$ and $0 \%$ (for number $\geq 4$ ), respectively $(P=0.000)$ (Fig. 2$)$. These analytic data clearly indicate that the patients with 2 to 3 tumor number survived better with much lower recurrence rates than those with tumor number $\geq 4$.

\section{Relationship between recurrence and tumor size}

On the basis of the cumulative tumor size, patients were divided into two groups, $\leq 5 \mathrm{~cm}$ (45 cases) and $>5 \mathrm{~cm}$ (33 cases). Kaplan-Meier analysis was performed to analyze the DFS for the patients in the two groups. The 1-, 2- and 5-year DFS rates were $70.6,50.1$ and $22 \%$ for the $\leq 5 \mathrm{~cm}$ group, and $51.5,18.2$ and $9.1 \%$ for the $>5 \mathrm{~cm}$ group, respectively $(P=$ 0.009 , Fig. 3), indicating that the patients with cumulative tumor size $\leq 5$ but $>3 \mathrm{~cm}$ showed significant lower recurrence rates than those with cumulative tumor size $>5 \mathrm{~cm}$.

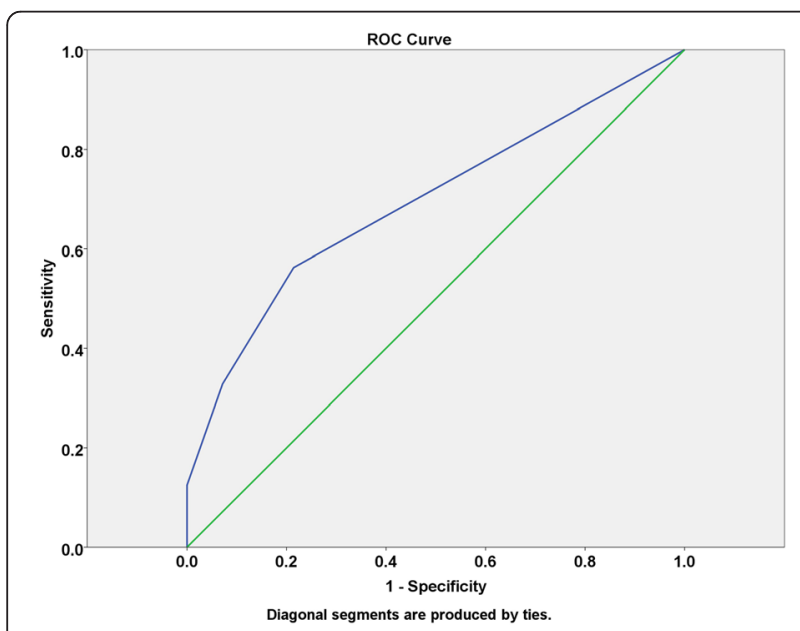

Fig. 1 ROC curves of tumor number and recurrence

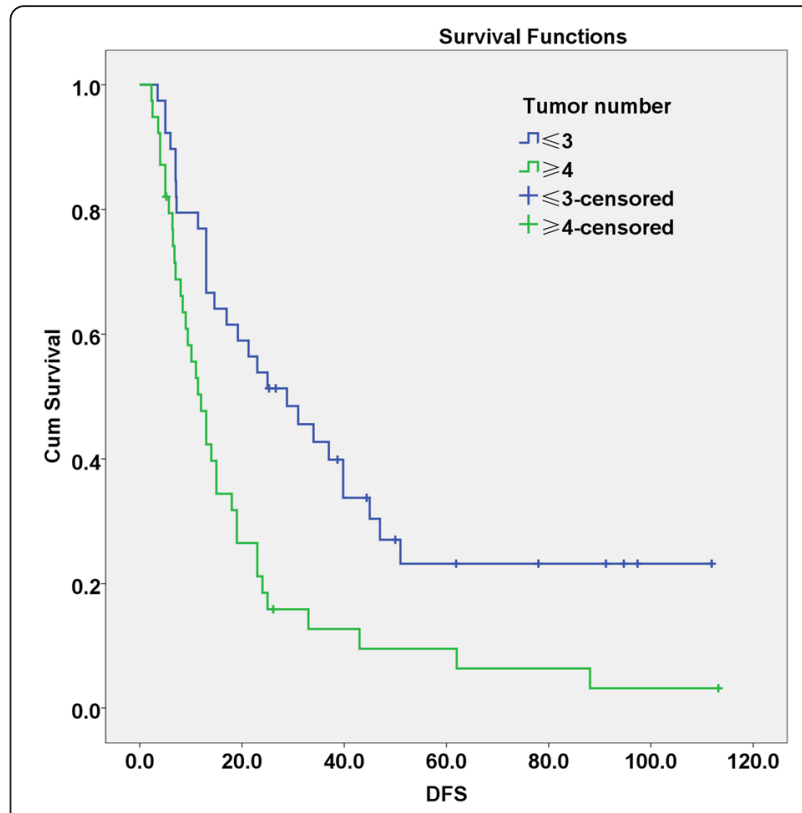

Fig. 2 Disease-free survival (DFS) curves of tumor number $\leq 3$ and $\geq 4$. The upper curve represents DFS of patients with $\leq 3$ tumors, while the lower curve represents DFS of patients with $\geq 4$ tumors

\section{Comparison of DFS and OS between high-risk and non-high-risk groups}

Patients with tumor number $\geq 4$ and cumulative tumor size $>5 \mathrm{~cm}$ were classified as the high-risk group (42 cases) while the other 36 patients as the non-high-risk group. Kaplan-Meier survival analysis was performed to

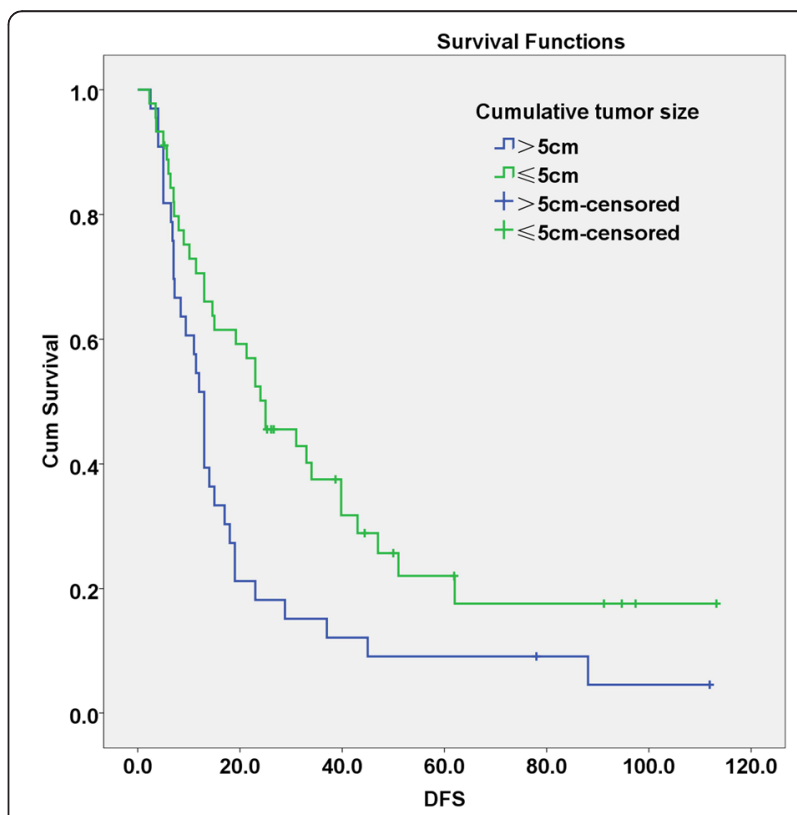

Fig. 3 Disease-free survival (DFS) curves of cumulative tumor size $\leq 5 \mathrm{~cm}$ and $>5 \mathrm{~cm}$. The upper curve represents DFS of patients with a cumulative tumor size of $\leq 5.0 \mathrm{~cm}$, while the lower curve represents DFS of patients with a cumulative tumor size $>5.0 \mathrm{~cm}$ 
analyze the DFS. For patients in the high-risk and nonhigh-risk groups, the 1-, 2-, and 5-year DFS rates were 49, 17.2 and 7.4 (for high-risk), and 77.8, 58.3 and $27.2 \%$ (for non-high-risk), respectively $(P=0.001)$; the OS rates at 1, 2. and 5 years were 78.6, 54.8 and $13.4 \%$ (for high-risk), and 94.4, 83.3 and $51.8 \%$ (for nonhigh-risk), respectively $(P=0.000)$ (Fig. 4$)$. The significance of the analytic results was in line with those in 3.2 and 3.3 sections, i.e., the recurrence rates of the non-high-risk group were significantly lower than those of the high-risk group.

\section{Discussion}

Tumor recurrence after resection is an important factor affecting the prognosis of patients with HCC. The BCLC staging system was proposed in 1999 by Llovet et al. [8] and modified by the American Association for the Study of Liver Diseases in 2005 [9]. This staging system
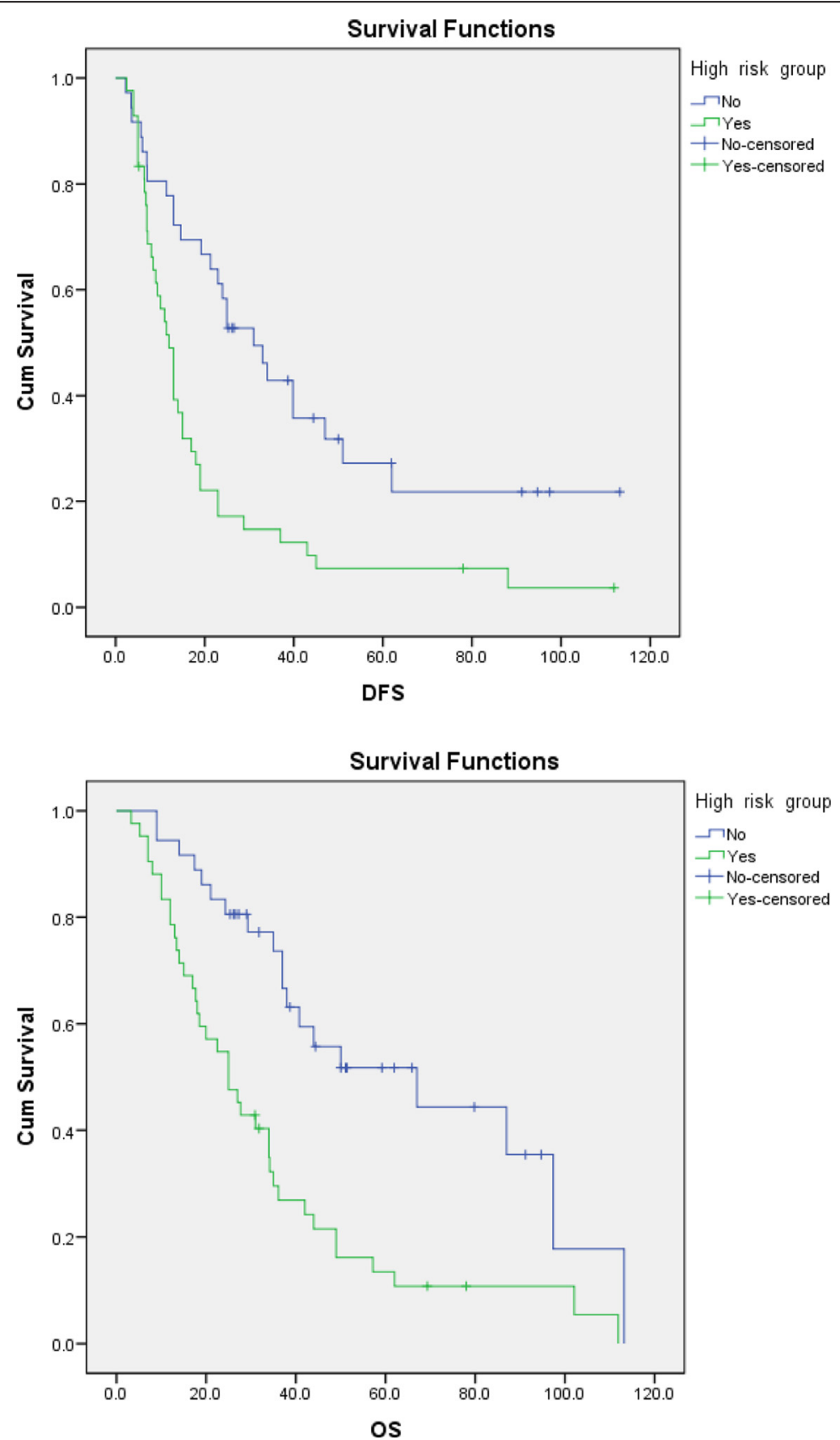

Fig. 4 Disease-free survival (DFS) and overall survival (OS) curves of high-risk and non-high-risk group. The upper curves represent the DFS and OS of the non-high-risk group, while the lower curves are DFS and OS of the high-risk group 
comprehensively considers tumor size and number, vascular invasion, liver function, and general condition. It has been accepted worldwide at present. The system recommends that patients with stage B HCC should be treated with TACE. However, many studies have indicated that for patients with BCLC stage B HCC, the prognosis for surgical resection treatment is superior to that for TACE. For example, from 171 patients with BCLC stage B HCC, Lin [5] found that the 1-, 2- and 3year OS rates after surgical resection or TACE was 83, 62 and $49 \%$ or 39,5 and $2 \%$, respectively. The prognosis of surgical resection was obviously better than that of TACE.

Considering that patients with BCLC stage B HCC frequently have multiple tumors with or without vascular invasion and intrahepatic metastasis, we think that it is necessary to explore further the indicators of surgical resection for this stage HCC to obtain a better prognosis. In this study, we identified that tumor number $\geq 4$ and cumulative tumor size $>5 \mathrm{~cm}$ were independent risk factors influencing the prognosis of BCLC stage B HCC after R0 liver resection. Median DFS and OS in patients with these two factors were 12.0 and 25.0 months, respectively, which were significantly lower than those of patients with tumor number 2 or 3 and cumulative tumor size $\leq 5 \mathrm{~cm}$ (31.0 and 67.0 months, $P<0.01$ ). In keeping with our current study, many studies have proved that tumor number [10-14] and tumor size $[10,11,15-17]$ can affect prognosis after resection of HCC. For example, Shah et al. [10] discovered that multiple tumors and large tumor size are independent risk factors of postoperative recurrence of HCC.

It is clinically significant that tumor number and size have been identified as two independent risk factors. The blood supply of HCC is mainly from the hepatic artery and portal vein. Therefore, large tumors always have a more abundant blood supply, grow faster, break through the capsule more easily and transfer to and infiltrate the surrounding liver tissue readily. Large tumors also more easily cause invasion of the portal vein, increasing the probability of postoperative intrahepatic recurrence. Multiple tumors in the liver are mostly due to intrahepatic metastasis, and an increase in tumor number usually indicates more rapid metastasis. For these patients, even if the visible tumors are totally removed, small metastases may also be present in the liver, resulting in recurrence of HCC, which is consistent with the finding of this study that patients with $\mathrm{HCC}$ stage $\mathrm{B}$ with $\geq 4$ tumors or a cumulative tumor size of $>5.0 \mathrm{~cm}$ had higher postoperative recurrence rates.

Our study was limited by the small sample size and regional area, which may not be representative of patients with BCLC stage B HCC in other areas. Moreover, the follow-up period for some patients was relatively short. Nonetheless, these patients were included in the analysis because they meet the BCLC stage B-staging standard, although they had many significant risk factors reported already, e.g., age of $>60$ years; 4-5 nodules; tumor size of $>5.0 \mathrm{~cm}$; significant amount of perioperative blood loss; and much higher AFP, ALT, and GGT levels than normal. In spite of these limitations, the results of this study revealed that both tumor number and tumor size were the main staging indicators of BCLC stage B HCC. Therefore, our study has important significance in estimating clinical treatment and predicting the prognosis to guide postoperative treatment.

\section{Conclusions}

This retrospective analysis suggests that patients with BCLC stage B HCC having two or three tumors and a cumulative tumor size of $>3.0 \mathrm{~cm}$ but $\leq 5.0 \mathrm{~cm}$ achieved better outcomes from R0 surgical resection than other patients with stage B HCC.

Competing interests

The authors declare that they have no competing interests.

\section{Authors' contributions}

WX collected and analyzed the data. WZ collected and analyzed the data. WL collected the data and wrote the manuscript. All authors read and approved the final manuscript.

\section{Acknowledgments}

We thank Dr. Zhiqiang Qu (Center for Medical Research, Affiliated Hospital of Qingdao University) for critical revision and comments on the manuscript. This project was supported by the Funding for Excellent Departments (Qingdao University) to WL.

Received: 9 August 2015 Accepted: 8 April 2016

Published online: 19 April 2016

\section{References}

1. Marrero JA. Multidisciplinary management of hepatocellular carcinoma: where are we today? Semin Liver Dis. 2013;33(S1):S3-10.

2. Ferlay J, Shin HR, Bray F, et al. Estimates of worldwide burden of cancer in 2008: GLOBOCAN 2008. Int J Cancer. 2010;127(12):2893-917.

3. Bruix J, Llovet JM. Prognostic prediction and treatment strategy in hepatocellular carcinoma. Hepatology. 2002;35(3):519-24.

4. Torzilli G, Belghiti J, et al. A snapshot of the effective indications and results of surgery for hepatocellular carcinoma in tertiary referral centers: is it adherent to the EASL/AASLD recommendations?: an observational study of the HCC East-west study group. Ann Surg. 2013;257(5):929-37.

5. Lin CT, Hsu KF, et al. Comparing hepatic resection andtranscatheterarterial chemoembolization for Barcelona Clinic Liver Cancer (BCLC) stage B hepatocellular carcinoma: change for treatment of choice? World J Surg. 2010;34(9):2155-61.

6. Wang JH, Chang CS, Hu TH, et al. The efficacy of treatment schedules according to Barcelona clinic liver cancer staging for hepatocellular carcinoma: survival analysis of 389 patients. Eur J Cancer. 2008:44(7):1000-6.

7. Jianyong $L$, Lunan $Y$, et al. Barcelona clinic liver cancer stage $B$ hepatocellular carcinoma: transcatheterarterial chemoembolization or hepatic resection? Medicine. 2014;93(26):180.

8. Llovet JM, Bru C, Bruix J. Prognosis of hepatocellular carcinoma: the BCLC staging classification. Semin Liver Dis. 1999;19(3):329-38.

9. Bruix J, Sherman M. Management of hepatocellular carcinoma. Hepatology. 2005;42(5):1208-36.

10. Shah SA, Cleary SP, et al. Recurrence after liver resection for hepatocellular carcinoma: risk factors, treatment, and outcomes. Surgery. 2007;141(3):330-9. 
11. Cha C, Fong $Y$, et al. Predictors and patterns of recurrence after resection of hepatocellular carcinoma. J Am Coll Surg. 2003;197(5):753-8.

12. Ruzzenente A, Capra F, Pachera S, et al. Is liver resection justified in advanced hepatocellular carcinoma? Results of an observational study in 464 patients. J Gastrointest Surg. 2009;13(7):1313-20.

13. Nojiri $\mathrm{K}$, Tanaka $\mathrm{K}$, et al. The efficacy of liver resection for multinodular hepatocellular carcinoma. Anticancer Res. 2014;34(5):2421-6.

14. Jun $\mathrm{CH}$, da Sim W, et al. Risk factors for patients with stage IVB hepatocellular carcinoma and extension into the heart: prognostic and therapeutic implications. Yonsei Med J. 2014;55(2):379-86.

15. Yip VS, Gomez D. Tumour size and differentiation predict survival after liver resection for hepatocellular carcinoma arising from non-cirrhotic and nonfibrotic liver: a case-controlled study. Int J Surg. 2013;11(10):1078-82.

16. Zhou L, Liu C, Meng FD, Kai Q, Tian F, Tai MH, Wei J-C, Wang R-T. Long-term prognosis in hepatocellular carcinoma patients after hepatectomy. Asian Pacific J Cancer Prev. 2012;13(2):483-6.

17. Faber $\mathbf{W}$, Siamak $\mathrm{S}$, et al. Long-term results of liver resection for hepatocellular carcinoma in noncirrhotic liver. Surgery. 2013;153(4):510-7.

Submit your next manuscript to BioMed Central and we will help you at every step:

- We accept pre-submission inquiries

- Our selector tool helps you to find the most relevant journal

- We provide round the clock customer support

- Convenient online submission

- Thorough peer review

- Inclusion in PubMed and all major indexing services

- Maximum visibility for your research

Submit your manuscript at www.biomedcentral.com/submit
Biomed Central 